\title{
Negative permeability due to exchange spin-wave resonances in thin magnetic films with surface pinning
}

\author{
R. V. Mikhaylovskiy, ${ }^{*}$ E. Hendry, and V. V. Kruglyak ${ }^{\dagger}$ \\ School of Physics, University of Exeter, Stocker Road, Exeter EX4 4QL, United Kingdom \\ (Received 6 June 2010; revised manuscript received 27 September 2010; published 30 November 2010)

\begin{abstract}
We report a theory of the effective permeability of multilayered metamaterials containing thin ferromagnetic layers with magnetization pinned on either one or both surfaces. Because of the pinning and small film thickness, the lowest frequency magnetic resonances are due to nonuniform exchange spin waves with frequencies far above those expected for uniform ferromagnetic resonance in known magnetic materials. Yet, the coupling of the nonuniform spin-wave modes to the electromagnetic field is shown to be strong enough to lead, for magnetic parameters characteristic for conventional transition metal alloys, to negative values of the effective permeability at frequencies of several hundred gigahertzs. The permittivity of metals is already negative in this frequency range. Hence, this system represents a negative refractive index metamaterial at subterahertz frequencies. The ways by which to maximize the frequency and the strength of the negative magnetic response are analyzed.
\end{abstract}

DOI: $10.1103 /$ PhysRevB.82.195446

PACS number(s): 78.67.Pt, 75.78. $-\mathrm{n}$

\section{INTRODUCTION}

Electromagnetic metamaterials gain their properties from their structure rather than atomic composition and can be engineered to provide electromagnetic properties that are not readily available in nature. Normally, this involves fabrication of arrays of intricate artificial elements (meta-atoms) with size small as compared to the wavelength of the electromagnetic waves (light) of interest. So, for propagating light, such arrays behave as continuous media with some effective values of the permittivity, permeability, and refractive index. These effective properties originate from the averaging of and hence can be tailored by tuning the electromagnetic responses of the constituent elements. In this way, it is thought that metamaterials might lead to creation of such unusual objects and effects as the perfect lens ${ }^{1,2}$ and electromagnetic cloaking. ${ }^{3}$

One of the most exciting demonstrations of the concept has been the development of negative refractive index metamaterials. To obtain the negative refractive index, one has to find a frequency range in which the real parts of both permittivity and permeability are simultaneously negative, although this is not a rigorously necessary condition. ${ }^{4} \mathrm{Nega-}$ tive permittivity is relatively easy to achieve since all metals exhibit negative permittivity at frequencies well below their plasma frequency. However, negative permeability is more difficult to achieve since the coupling of the magnetic field of light to atoms is much weaker than the coupling of the corresponding electric field. One can overcome the problem of weak coupling to the ac magnetic field by using such structures as split ring resonators (SPRs), which can produce a diamagnetic response and hence negative permeability above a certain resonance frequency determined by the diameter of the split ring. ${ }^{5}$ This concept was initially demonstrated in experiments at microwave frequencies. ${ }^{6,7}$ However, to resonate at terahertz $(\mathrm{THz})$ and optical frequencies, SPRs must have nanoscale sizes and hence are harder to fabricate. Moreover, such structures are very lossy at high frequencies, leading to low figures of merit. ${ }^{4}$ Other schemes for generating negative permeability, e.g., those employing U-shaped elements ${ }^{8}$ or arrays of holes, ${ }^{9}$ suffer from similar drawbacks. The search for new ways to create negative permeability is therefore ongoing.

Another way of achieving negative permeability was first pointed out already by Veselago, the discoverer of the phenomenon of negative refraction. ${ }^{1}$ The idea is to utilize the negative permeability existing in resonant magnetic gyrotropic materials. ${ }^{10}$ However, bulk ferromagnets normally exhibit gigahertz $(\mathrm{GHz})$ resonant frequencies (see, e.g., Ref. 11), thereby restricting their application in metamaterials to the same low $\mathrm{GHz}$ frequency range. At the same time, it is known that spin waves (nonuniform waves of precessing magnetization) can have frequencies reaching several THzs in the exchange dominated regime, ${ }^{12-14}$ although their coupling to uniform electromagnetic field has been traditionally considered weak. In particular, exchange spin waves come into play in studies of the high-frequency magnetic response of composites containing magnetic inclusions of cylindrical ${ }^{15-17}$ and spherical ${ }^{18-22}$ shapes.

Here, we show that the coupling of the electromagnetic field to exchange spin waves confined in thin ferromagnetic films with surface pinning can be sufficiently strong to result in negative values of the magnetic permeability at frequencies of several hundred GHzs, with a potential to reach $\mathrm{THz}$ range. The metallic magnetic films assumed in our calculations have negative permittivity at $\mathrm{THz}$ and sub- $\mathrm{THz}$ frequencies, and so, no additional elements are required to obtain negative dielectric response. Hence, we suggest that such films can be used to construct negative index metamaterials. We analyze the dependence of the strength and frequency of the observed negative permeability upon properties of the magnetic films.

The theory and measurement of resonant magnetic permeability are traceable back to the onset of magnetism research. ${ }^{23-25}$ The use of uniform high-frequency magnetic field to excite nonuniform spin waves in thin films was demonstrated already by Kittel, ${ }^{26}$ who pointed out the importance of the spin pinning at the films' surfaces. Significant efforts 


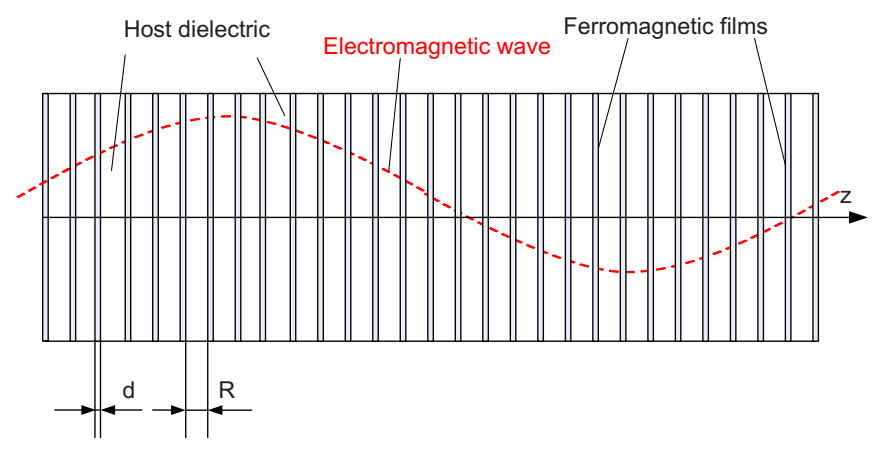

FIG. 1. (Color online) The sketch of the proposed metamaterial is schematically shown. Thin ferromagnetic layers are separated by layers of the host dielectric. The electromagnetic wave (light) propagates along $z$ direction. The light wavelength is much greater than the characteristic dimensions of the superlattice.

were applied to elucidating physics of ferromagnetic resonance measurements of thick magnetic films, notably of eddy current and exchange-conductivity contributions to the resonance frequency and linewidth in metals. ${ }^{27-31}$ Subsequent studies concentrated on various magnetic thin films and multilayers, ${ }^{32-37}$ with a focus on the spectrum of their spinwave resonances. Here, we present a theory of the highfrequency permeability resulting from such resonances in very thin films with surface pinning and its application to the issue of designing the negative effective permeability.

\section{THEORETICAL FORMALISM}

The structure of interest is schematically depicted in Fig. 1. To find its electromagnetic properties one has to solve Maxwell equations together with Landau-Lifshitz equations for each film, provided that the permittivity of the films is known. Generally, the electromagnetic and micromagnetic aspects of the problem are characterized by very different length scales. This allows us to simplify the calculation by neglecting the exchange-conductivity and eddy current effects and then by using the effective medium concept. Indeed, if the wavelength of light is long enough compared to the period of the superlattice, the magnetic field of light can be considered uniform across each particular layer or even across several consecutive periods of the structure. So, neglecting magnetodipole interaction between magnetic films, we can find the dynamic response of the magnetization of a single film to a given uniform ac magnetic field, and then calculate the response of the superlattice as an effective medium.

We consider cases of in-plane and out-of-plane magnetized films. We derive general formulas describing standing spin waves in the films and average the magnetic response to obtain the tensor of macroscopic effective permeability of the metamaterial. Then we consider propagation of light through the material described by the calculated tensor. We show that, for certain geometries, it is possible to describe normal waves' propagation using scalar refractive indexes.

\section{A. Geometry of the problem and basic equations}

We begin by considering an array of parallel thin ferromagnetic films (with thickness $d \sim 1-10 \mathrm{~nm}$ ) embedded in a
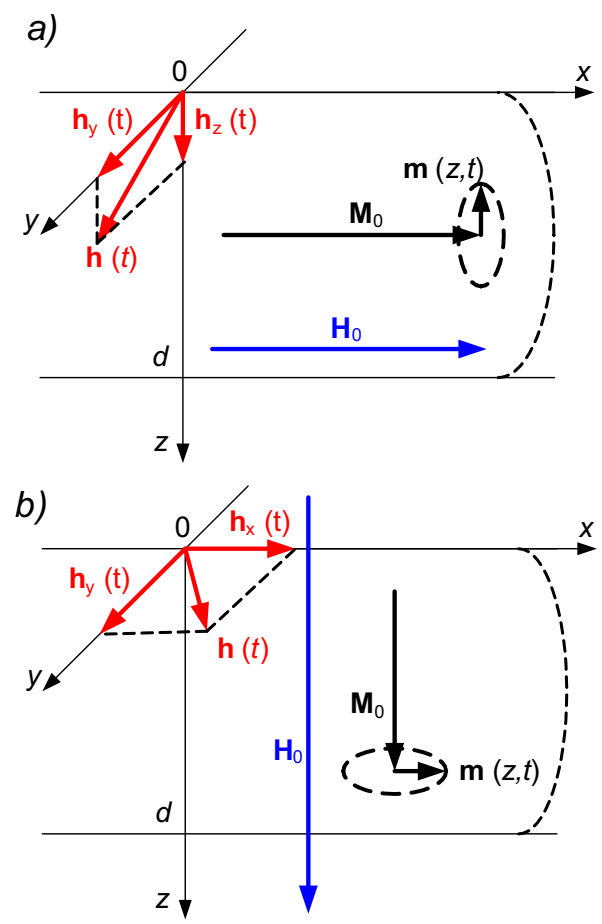

FIG. 2. (Color online) The magnetic geometry of the ferromagnetic layers is shown for the (a) in-plane and (b) out-of-plane magnetizations.

nonmagnetic host material (Fig. 1). The films are separated by distance $R$, which is assumed large enough to neglect any interaction between the films. In this model, the films play a role of meta-atoms. The wavelength of light is assumed to be much greater than both the thickness of and the distance between the films. Hence, the magnetic field of the light is considered to be uniform over many consecutive layers of the metamaterial. The length and width of the structure are assumed to be much greater than both the thickness of the film and the transverse size of the propagating light beam. This allows us to consider this structure as one-dimensional metamaterial.

Let us study spin waves excited by an alternating magnetic field in a single ferromagnetic film magnetized by a static bias magnetic field (Fig. 2). The orientation of the Cartesian coordinate system is chosen so that its $z$ axis is perpendicular to the film surface. Then, we can assume that all physical values and parameters considered below are functions of $z$ but not of $x$ and $y$.

To describe the motion of magnetization $\mathbf{M}$ we use the Landau-Lifshitz-Gilbert equation ${ }^{11,23}$ (note that all formulas in this paper are in CGS units),

$$
\frac{\partial \mathbf{M}}{\partial t}=-\gamma\left[\mathbf{M} \times \mathbf{H}_{e f f}\right]+\frac{\nu}{M}\left[\mathbf{M} \times \frac{\partial \mathbf{M}}{\partial t}\right],
$$

where $\gamma$ is the absolute value of the gyromagnetic ratio, $\nu$ is the Gilbert damping constant, and the effective magnetic field is $\mathbf{H}_{e f f}=\mathbf{H}_{0}+\mathbf{H}_{e x}+\mathbf{h}+\mathbf{h}_{d m}$. Here, $\mathbf{H}_{0}$ is the internal static magnetic field, $\mathbf{H}_{e x}=\alpha \Delta \mathbf{M}$ is the exchange field with $\alpha$ being the exchange constant, $\mathbf{h}$ is the external dynamic magnetic field (i.e., the magnetic field of light), and $\mathbf{h}_{d m}$ is the dynamic 
demagnetizing field. We are interested in magnetic excitations that are small compared to the equilibrium magnetization $\mathbf{M}_{0}$. We therefore introduce a vector of the magnetization in the form $\mathbf{M}=\mathbf{M}_{0}+\mathbf{m}(z, t)$, where $\left|\mathbf{M}_{0}\right| \gg|\mathbf{m}|$ and linearize Eq. (1) with respect to terms smallest in $\mathbf{m}$ and $\mathbf{h}$. The demagnetizing field is determined by the magnetostatic equations $\nabla \times \mathbf{h}_{d m}=0$ and $\nabla \cdot\left(\mathbf{h}_{d m}+4 \pi \mathbf{m}\right)=0$. Since all variables are assumed here independent from $x$ and $y$ coordinates, $\mathbf{h}_{d m}=-4 \pi \mathbf{z}_{0}\left(\mathbf{m} \cdot \mathbf{z}_{0}\right)$. The justification for neglecting the exchange-conductivity effect and hence using equation $\nabla$ $\times \mathbf{h}_{d m}=0$ is given in Appendix A.

Let us consider two different geometries shown in Fig. 2: an in-plane magnetization, when vectors $\mathbf{H}_{0}$ and $\mathbf{M}_{0}$ are parallel to the $x$ axis, and an out-of-plane magnetization, when vectors $\mathbf{H}_{0}$ and $\mathbf{M}_{0}$ are parallel to the $z$ axis. Due to the shape anisotropy, the former geometry is realized in experiments more frequently while the latter is more popular among theoreticians due to the easier account of the magnetodipole fields. So, the surface impedance and permeability for perpendicularly magnetized films with surface pinning was studied, e.g., in Refs. 34, 38, and 39. Here, both geometries are considered within the same formalism for the sake of completeness and comparison.

Projecting the linearized Eq. (1) to the coordinate system we obtain in the first case (the $x$ component of $\mathbf{h}$ is collinear with and hence does not couple to $\mathbf{M}_{0}$ )

$$
\begin{gathered}
\frac{\partial m_{y}}{\partial t}=\gamma \alpha M_{0} \frac{\partial^{2} m_{z}}{\partial z^{2}}-\gamma H_{0} m_{z}-\nu \frac{\partial m_{z}}{\partial t}-4 \pi \gamma M_{0} m_{z}+\gamma M_{0} h_{z}, \\
\frac{\partial m_{z}}{\partial t}=-\gamma \alpha M_{0} \frac{\partial^{2} m_{y}}{\partial z^{2}}+\gamma H_{0} m_{y}+\nu \frac{\partial m_{y}}{\partial t}-\gamma M_{0} h_{y} .
\end{gathered}
$$

In the second case, the $z$ component of $\mathbf{h}$ is collinear with $\mathbf{M}_{0}$, and so, the corresponding linearized system of equations is given by

$$
\begin{gathered}
\frac{\partial m_{x}}{\partial t}=\gamma \alpha M_{0} \frac{\partial^{2} m_{y}}{\partial z^{2}}-\gamma H_{0} m_{y}-\nu \frac{\partial m_{y}}{\partial t}+\gamma M_{0} h_{y}, \\
\frac{\partial m_{y}}{\partial t}=-\gamma \alpha M_{0} \frac{\partial^{2} m_{x}}{\partial z^{2}}+\gamma H_{0} m_{x}+\nu \frac{\partial m_{x}}{\partial t}-\gamma M_{0} h_{x} .
\end{gathered}
$$

Here and in the following, indexes " $a$ " and " $b$ " in the equations' numbering correspond to the cases of in-plane and out-of-plane magnetizations, respectively.

\section{B. General solution of the microscopic magnetization}

The dynamic magnetic field $\mathbf{h}$ is a function of time, and we are interested in the forced oscillations of the system. Applying the Fourier transform with respect to time to Eqs. (2a) and (2b), we obtain in frequency domain (symbol $\sim$ denotes Fourier images)

$$
i \Omega \tilde{m}_{y}=\frac{\partial^{2} \tilde{m}_{z}}{\partial \xi^{2}}-\eta \tilde{m}_{z}-i \Omega \nu \tilde{m}_{z}-4 \pi \tilde{m}_{z}+\tilde{h}_{z}
$$

$$
i \Omega \tilde{m}_{z}=-\frac{\partial^{2} \tilde{m}_{y}}{\partial \xi^{2}}+\eta \tilde{m}_{y}+i \Omega \nu \tilde{m}_{y}-\tilde{h}_{y},
$$

and

$$
\begin{gathered}
i \Omega \tilde{m}_{x}=\frac{\partial^{2} \tilde{m}_{y}}{\partial \xi^{2}}-\eta \tilde{m}_{y}-i \Omega \nu \tilde{m}_{y}+\tilde{h}_{y}, \\
i \Omega \tilde{m}_{y}=-\frac{\partial^{2} \tilde{m}_{x}}{\partial \xi^{2}}+\eta \tilde{m}_{x}+i \Omega \nu \tilde{m}_{x}-\tilde{h}_{x} .
\end{gathered}
$$

The dimensionless variables, coefficients, and parameters are given by

$$
\Omega=\frac{\omega}{\gamma M_{0}}, \quad \eta=\frac{H_{0}}{M_{0}}, \quad \xi=\frac{z}{\sqrt{\alpha}} .
$$

The dispersion law of Eqs. (3a) and (3b) is

$$
\begin{gathered}
\Omega^{2}=\left(\eta+4 \pi+K_{\perp}^{2}+i \Omega \nu\right)\left(\eta+K_{\perp}^{2}+i \Omega \nu\right), \\
\Omega^{2}=\left(\eta+K_{\|}^{2}+i \Omega \nu\right)^{2} .
\end{gathered}
$$

Here $K_{\perp}$ and $K_{\|}$denote the wave numbers of spin waves in the cases of in-plane and out-of-plane magnetizations, respectively. To avoid confusion, we remind the reader that the corresponding wave vectors are perpendicular to the plane of the layer, i.e., the magnetization dynamics excited in the considered geometries are uniform in the plane of the layers. Rearranging Eqs. (5a) and (5b) gives

$$
\begin{gathered}
K_{\perp 1}^{2}=k_{\perp}^{2}=\sqrt{\Omega^{2}+4 \pi^{2}}-(\eta+4 \pi+i \Omega \nu), \\
K_{\perp 2}^{2}=-\kappa_{\perp}^{2}=-\sqrt{\Omega^{2}+4 \pi^{2}}-(\eta+4 \pi+i \Omega \nu)
\end{gathered}
$$

and

$$
\begin{gathered}
K_{\| 1}^{2}=k_{\|}^{2}=\Omega-(\eta+i \Omega \nu), \\
K_{\| 2}^{2}=-\kappa_{\|}^{2}=-\Omega-(\eta+i \Omega \nu) .
\end{gathered}
$$

The full solution of Eqs. (3a) and (3b) is given by the sum of the general solution of the corresponding homogeneous system and partial solution of the inhomogeneous one. This solution has the following form:

$$
\begin{aligned}
\tilde{m}_{y}= & C_{1} e^{i k_{\perp} \xi}+C_{2} e^{-i k_{\perp} \xi}+C_{3} e^{\kappa_{\perp} \xi}+C_{4} e^{-\kappa_{\perp} \xi}+\tilde{f}_{\perp} \tilde{h}_{y}+\tilde{p}_{\perp} \tilde{h}_{z}, \\
\tilde{m}_{z}= & \frac{\eta+i \Omega \nu+k_{\perp}^{2}}{i \Omega}\left(C_{1} e^{i k_{\perp} \xi}+C_{2} e^{-i k_{\perp} \xi}\right) \\
& +\frac{\eta+i \Omega \nu-\kappa_{\perp}^{2}}{i \Omega}\left(C_{3} e^{\kappa_{\perp} \xi}+C_{4} e^{-\kappa_{\perp} \xi}\right)+\tilde{g}_{\perp} \tilde{h}_{y}+\tilde{q}_{\perp} \tilde{h}_{z}
\end{aligned}
$$

for the coupled Eq. (3a) and

$$
\tilde{m}_{x}=D_{1} e^{i k_{\|} \xi}+D_{2} e^{-i k_{\|} \xi}+D_{3} e^{\kappa_{\|} \xi}+D_{4} e^{-\kappa_{\|} \xi}+\tilde{f}_{\|} \tilde{h}_{y}+\tilde{p}_{\|} \tilde{h}_{x},
$$




$$
\begin{aligned}
\widetilde{m}_{y}= & \frac{\eta+i \Omega \nu+k_{\|}^{2}}{i \Omega}\left(D_{1} e^{i k_{\|} \xi}+D_{2} e^{-i k_{\|} \dot{\xi}}\right) \\
& +\frac{\eta+i \Omega \nu-\kappa_{\|}^{2}}{i \Omega}\left(D_{3} e^{\kappa_{\|} \xi}+D_{4} e^{-\kappa_{\|} \dot{\xi}}\right)+\widetilde{g}_{\|} \widetilde{h}_{y}+\widetilde{q}_{\|} \widetilde{h}_{x}
\end{aligned}
$$

for the coupled Eq. (3b). Here $\widetilde{f}_{\perp, \|,}, \widetilde{p}_{\perp, \|}, \widetilde{g}_{\perp, \|}$, and $\widetilde{q}_{\perp, \|}$ make up the partial solution, are independent from $\xi$ (see Appendix B), and describe the uniform ferromagnetic resonance. The amplitudes $C_{1}, C_{2}, C_{3}, C_{4}$ and $D_{1}, D_{2}, D_{3}, D_{4}$ are found from the boundary conditions at the interfaces of the film. We use the exchange boundary conditions ${ }^{11}$

$$
\begin{gathered}
-\frac{\partial \widetilde{m}_{i}}{\partial \xi}+\beta_{1} \widetilde{m}_{i}=0, \quad \xi=0, \\
\frac{\partial \widetilde{m}_{i}}{\partial \xi}+\beta_{2} \widetilde{m}_{i}=0, \quad \xi=l,
\end{gathered}
$$

Here, $l=\frac{d}{\sqrt{\alpha}}$ is dimensionless film thickness while $\beta_{1}$ and $\beta_{2}$ are the pinning parameters at different surfaces of the film. They describe the strength of the effective magnetic field $H_{p i n}$ at the surfaces due to spin pinning. This effective field might refer to the surface anisotropy or exchange bias at the interface between ferromagnet and antiferromagnet. The pinning parameter is defined as

$$
\beta=\frac{H_{p i n}}{M_{0}} .
$$

Substituting Eqs. (7a) and (7b) into Eq. (8) one can find the amplitudes. The corresponding formulas are very bulky and are therefore shown in Appendix B. The general form of these formulas is

$$
\begin{gathered}
C_{j}=a_{\perp j}(\Omega) \tilde{h}_{y}+b_{\perp j}(\Omega) \tilde{h}_{z}, \quad j=1,2,3,4, \\
D_{j}=a_{\| j}(\Omega) \tilde{h}_{x}+b_{\| j}(\Omega) \tilde{h}_{y}, \quad j=1,2,3,4,
\end{gathered}
$$

where $a_{\perp j}(\Omega), b_{\perp j}(\Omega), a_{\| j}(\Omega)$, and $b_{\| j}(\Omega)$ are functions of frequency. With the values of amplitudes $C$ and $D$ known, we have fully determined the excited microscopic magnetization $\mathbf{m}$.

\section{Permeability tensor}

With the solution for the microscopic magnetization at hand, we proceed to calculation of the magnetic susceptibility tensor. Due to its long wavelength, the light is insensitive to the fine structure of the spin-wave modes. So, one can integrate the microscopic magnetization over a physically infinitesimal (with respect to light wavelength $\lambda$ ) volume. In the one-dimensional case considered here, this is achieved by integrating over an interval with a length $\Lambda$, such that $d$ $<R \ll \Lambda \ll \lambda$. This gives us the macroscopic magnetization used in Maxwell equations together with permeability tensor $\hat{\mu}$. The associated procedure is described in Appendix C. Here, we only give the resulting effective permeability tensors,

$$
\hat{\mu}_{\perp}=\left(\begin{array}{ccc}
1 & 0 & 0 \\
0 & \mu_{\perp y} & i G_{\perp} \\
0 & -i G_{\perp} & \mu_{\perp z}
\end{array}\right),
$$

for the in-plane magnetized films and

$$
\hat{\mu}_{\|}=\left(\begin{array}{ccc}
\mu_{\|} & i G_{\|} & 0 \\
-i G_{\|} & \mu_{\|} & 0 \\
0 & 0 & 1
\end{array}\right),
$$

for the out-of-plane magnetized films. Although the effective permeability is a tensor, we show below that, in certain geometries, it is still possible to use scalar refractive indexes to describe the propagation of normal waves through the metamaterial.

The general form of the tensors given in Eqs. (11a) and (11b) is characteristic for so-called magnetogyrotropic media. ${ }^{11}$ Two types of normal waves exist in such a material —ordinary and extraordinary waves, each with different field components and polarization. Here, we consider the case of light propagating along $z$ direction, i.e., normal to the film plane. We assume the effective permittivity of the metamaterial to be a scalar quantity $\varepsilon$. Then, it is possible to describe the propagation of the normal waves in terms of the corresponding refractive indexes.

In the case of the in-plane magnetization and hence of light propagating perpendicular to the direction of the magnetization, the ordinary wave does not couple to spin waves since its magnetic field is parallel to the static magnetization. So, its propagation is described with refractive index $n_{\perp o}$ $=\sqrt{\varepsilon}$. In contrast, the extraordinary wave does interact with the magnetization. The refractive index of the extraordinary wave is given by $n_{\perp e}=\sqrt{\varepsilon\left(\mu_{\perp y}+\frac{G_{\perp}^{2}}{\mu_{\perp z}}\right)}$. Thus, one can expect the propagation of the extraordinary wave to be described by scalar permeability $\mu_{\perp e}=\mu_{\perp y}+\frac{G_{\perp}^{2}}{\mu_{\perp z}}$. When the magnetization is oriented along the direction of the electromagnetic wave propagation, the two normal waves are described by refractive indexes $n_{\| 1,2}=\sqrt{\varepsilon\left(\mu_{\|} \pm G_{\|}\right)}$. In this case, we can introduce two effective permeabilities: $\mu_{ \pm}=\left(\mu_{\|} \pm G_{\|}\right)$(see also Ref. 1). However, as discussed below, only $\mu_{+}$can reach negative values.

This concludes the mathematical formalism. In the next section, we discuss the feasibility of obtaining negative effective permeabilities at high frequencies.

\section{RESULTS AND DISCUSSION}

In this section, we apply the general theory developed above to the specific case of a superlattice with magnetic layers made of the $\mathrm{CoFe}$ alloys. $\mathrm{CoFe}$ has well-known material parameters while its technology is well developed due to applications in magnetic data storage devices. Importantly for the present study, it is characterized by some of the greatest values of the exchange constant and magnetization of saturation among transition metals and their alloys. In particular, we use the following values for magnetic parameters of the layers: $M_{0}=1700 \mathrm{emu} / \mathrm{cm}^{3}, \alpha=1.4 \times 10^{-12} \mathrm{~cm}^{2}$, and $\nu=0.01$. We assume that the filling fraction of the films in the structure is $\rho=0.25$. 


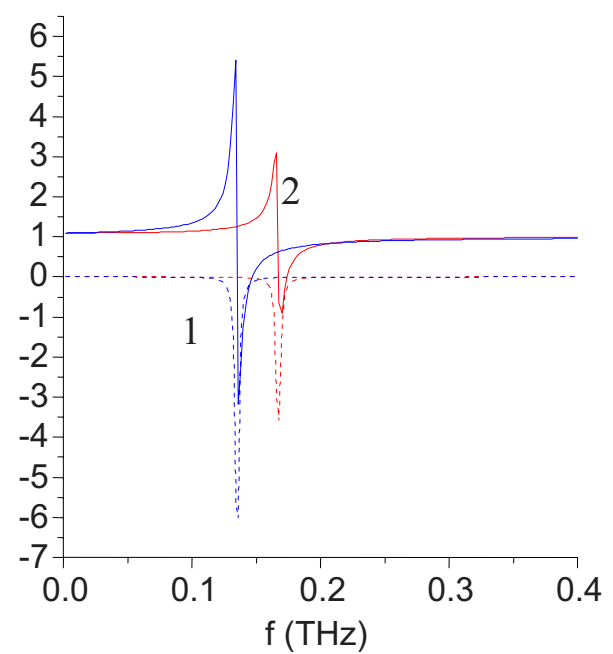

FIG. 3. (Color online) The calculated effective permeability is shown as a function of frequency for the cases of (1) in-plane and (2) out-of-plane magnetizations. The solid and dashed lines denote the real and imaginary parts of the effective permeability, respectively. The effective permeability is calculated for an array of CoFe films with thickness of $5 \mathrm{~nm}$. The filling fraction of 0.25 is assumed. The spins are perfectly pinned at one surface of the film and are free at the other.

Figure 3 shows the effective permeabilities calculated for the cases of in-plane and out-of-plane magnetizations by the bias magnetic field $H_{0}$ of $20 \mathrm{kG}$. One can clearly see from Fig. 3 that negative effective permeabilities exist in the region $0.1-0.2 \mathrm{THz}$. The bandwidth of these regions is around $20 \mathrm{GHz}$ in each case. The different resonant frequencies in the two cases arise from the different dispersion laws given by Eqs. (5a) and (5b).

The resistivity of CoFe films is typically $\sim 10^{14} \mathrm{~s}^{-1}$. In the frequency range considered here, such highly conducting materials are described by large permittivities: typically $|\varepsilon|$ $\sim 10^{3}-10^{4}$, Re $\varepsilon<0 .{ }^{40}$ One therefore expects a large negative effective permittivity response over a wide frequency range from a multilayered metamaterial containing CoFe layers. In addition, the material will exhibit negative refraction at frequencies where the effective permeability is also negative. The regions of negative refraction are determined by the resonances of standing spin waves in the magnetic layers.

We now turn our attention to optimization parameters of the structure in order to increase the strength of spin-wave resonances and to obtain higher resonance frequencies. One parameter that will affect the strength of resonance is damping. In order to optimize the interaction strength, one must seek ferromagnetic materials with the lowest spin-wave damping. The spin-wave resonance is also very sensitive to such parameters as the bias magnetic field, the pinning parameter, the magnetization of saturation, and the film thickness. In the discussion below, we address the roles of these parameters in the spin-wave resonance. Since the physical behavior of the effective permeability with respect to them is the same for in-plane and out-of-plane geometries, we consider only the dependence of $\mu_{\perp e}$, i.e., the case of in-plane magnetization.

The dependence of effective permeability $\mu_{\perp e}$ upon the frequency and dimensionless effective film thickness $l=\frac{d}{\sqrt{\alpha}}$ is

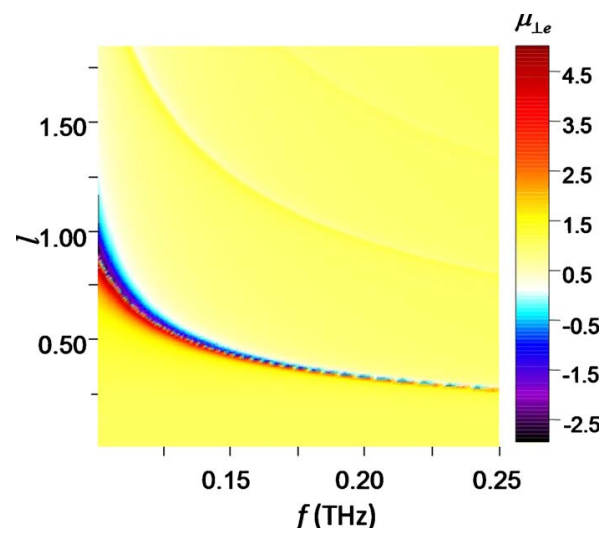

FIG. 4. (Color online) The effective permeability $\mu_{\perp e}$ is plotted as a function of the frequency and dimensionless thickness $l$, for the same assumptions and parameter values as in Fig. 3.

shown in Fig. 4. One can see that the frequency of the spinwave resonance can be increased by either decreasing the film thickness $d$ or by increasing the exchange constant $\alpha$. As expected, the strength of resonance increases as its frequency decreases.

Figure 5 shows effective permeability $\mu_{\perp e}$ as a function of the frequency and magnetization of saturation, with the other material parameters fixed. The resonance frequency increases as the value of the saturation magnetization increases. Also, the strength of the resonance increases due to the enhancement of the magnetic moment.

Figure 6 shows $\mu_{\perp e}$ as a function of the frequency and the pinning parameter $\beta$. For small pinning parameters, the resonance frequency is low but the resonance is strong. Increasing the pinning parameter increases the resonance frequency but somewhat decreases the resonance strength. Indeed, the increase in the pinning parameter decreases the wavelength of the excited spin waves and hence weakens their coupling to the uniform ac magnetic field. We estimate that an effective pinning field of approximately $1 \mathrm{~T}$ is required for a resonance in the $150-200 \mathrm{GHz}$ frequency range.

Of course, one can also increase the resonance frequency by increasing the bias magnetic field (Fig. 7). This can provide a convenient way to tune the negative refractive index

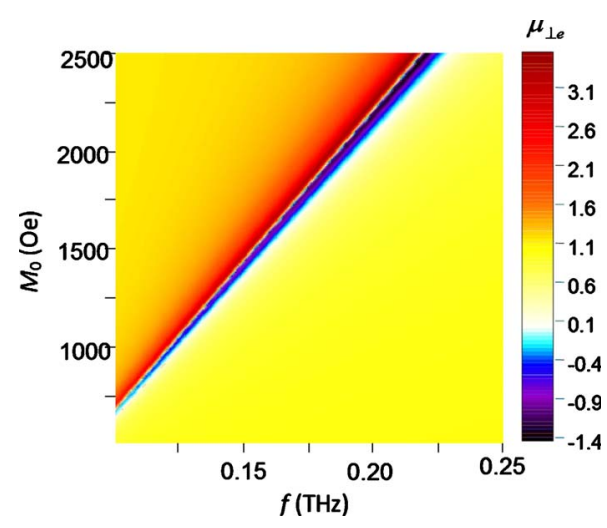

FIG. 5. (Color online) Effective permeability $\mu_{\perp e}$ is plotted as a function of the frequency and magnetization of saturation $M_{0}$, for the same assumptions and parameter values as in Fig. 3. 


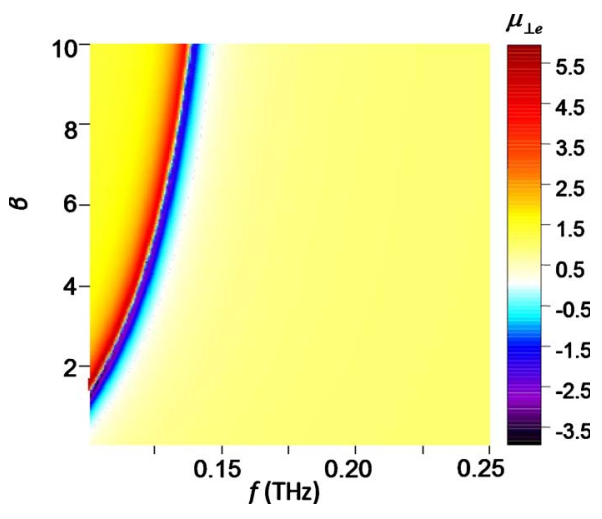

FIG. 6. (Color online) The effective permeability $\mu_{\perp e}$ is plotted as a function of frequency and pinning parameter $\beta$. The calculations are for the same assumptions and parameter values as in Fig. 3 , except the finite pinning strength assumed here.

frequency range of the proposed metamaterial.

Summarizing this part, to optimize the strength of magnetic resonance at sub- $\mathrm{THz}$ and $\mathrm{THz}$ frequencies one should use a ferromagnetic material with a large magnetization of saturation and low damping constant. By varying other parameters, such as the pinning parameter or the bias magnetic field, one can shift the resonance to higher frequencies but only at an expense of the resonance strength. However, for $\mathrm{CoFe}$ films, it appears impossible to obtain negative values of the effective permeability for frequencies greater than 200 $\mathrm{GHz}$. The strength of the resonance can be further increased by increasing the volume fraction of the magnetic material in which case the interaction between the different ferromagnetic films and associated magnonic behavior will need to be taken into account. ${ }^{13}$ This however is beyond the scope of this study.

Let us now discuss the results in the context of previous studies in the field of metamaterials. The sub- $\mathrm{THz}$ and $\mathrm{THz}$ metamaterials were intensively studied last years. ${ }^{41-45}$ Let us compare the figure of merit of the proposed magnonic metamaterial with those measured in or theoretically predicted for the other sub- $\mathrm{THz}$ and $\mathrm{THz}$ metamaterials. To estimate the figure of merit defined as $F=\left|\frac{\operatorname{Re} n}{\operatorname{Im} n}\right|$, we use the

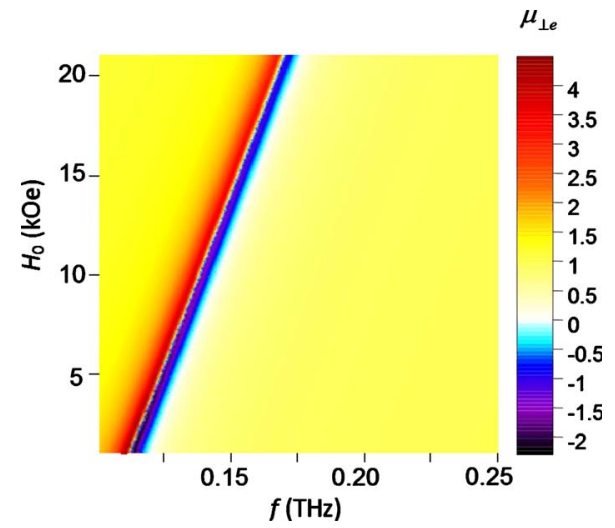

FIG. 7. (Color online) The effective permeability $\mu_{\perp e}$ is plotted as a function of the frequency and bias magnetic field $H_{0}$, for the same assumptions and parameter values as in Fig. 3 (spins are perfectly pinned at one side of the film). effective permittivity ${ }^{46} \varepsilon_{e f f}=\varepsilon_{h}+\rho \varepsilon_{\mathrm{CoFe}}$, where $\varepsilon_{h}$ and $\varepsilon_{\mathrm{CoFe}}$ are the permittivity of the host dielectric and $\mathrm{CoFe}$, respectively. $\varepsilon_{\mathrm{CoFe}} \sim(-1-i) \times 10^{3}-10^{4}$ (see, e.g., Ref. 40 ). Since $\varepsilon_{\mathrm{CoFe}} \gg \varepsilon_{h}$, one can neglect the contribution of the host dielectric material to the effective permittivity and treat the latter as $\varepsilon_{e f f} \approx \rho \varepsilon_{\mathrm{CoFe}}$. Thus the estimation of the figure of merit of the proposed metamaterial gives $F \sim 1-2$. This is on the order of figures of merits of other metamaterials in this frequency range. Higher figure of merits were also reported recently. ${ }^{47,48}$ However, the opportunities to improve magnonic metamaterials based on the proposed principle have not been exhausted. For example, nonmetallic antiferromagnets or ferrimagnets are promising candidates to replace the metallic ferromagnetic films with their high conductivity losses. ${ }^{49}$ On the other hand, the imaginary part of the permittivity and/or permeability plays an important role in the negative refraction effect. ${ }^{50}$

\section{CONCLUSIONS}

To conclude, we have proposed a so-called microwave magnonic metamaterial. The latter is based on an array of thin ferromagnetic films acting as resonators for exchange spin waves at high (sub-THz) frequencies. The coupling of the uniform magnetic field of the incident light to the nonuniform modes is facilitated by pinning of the spin waves at film surfaces. We have calculated the effective permeability tensor of the metamaterial for cases of in-plane and out-ofplane magnetizations. Our theory predicts negative values of the effective permeability in vicinity of the standing spinwave resonances. As compared to more conventional splitring resonators and similar structures, the proposed metamaterial might have the following advantages: (1) the negative permeability region is tunable by the bias magnetic field, (2) the gyrotropy allows one to manipulate the polarization of light, and (3) since the material is composed of continuous metallic elements, negative permittivity and, hence, negative refraction are also feasible. Moreover, since the frequency region of negative permeability is determined by the magnonic resonances of the component materials, there is an excellent outlook for finding and designing magnetic nanomaterials with stronger magnonic resonances at even higher frequencies.

\section{ACKNOWLEDGMENTS}

The research leading to these results has received funding from the European Community's Seventh Framework Programme (FP7/2007-2013) under Grant Agreement No. 228673 (MAGNONICS), from the Engineering and Physical Research Council (EPSRC) of the U.K., and from the Research Council of U.K. (RCUK). The authors also wish to thank R. J. Hicken and S. Foteinopoulou for useful comments.

\section{APPENDIX A: JUSTIFICATION OF EXCHANGE- CONDUCTIVITY EFFECTS NEGLECT}

Let us consider the relative importance of the exchangeconductivity and eddy current effects for the studied here 
system. To take them into account, one needs to replace the system of magnetostatic Maxwell equations with one including the Ohm and Faraday laws ${ }^{27}$

$$
\begin{gathered}
\nabla \cdot(\mathbf{H}+4 \pi \mathbf{M})=0, \\
\nabla \times \mathbf{E}=-\frac{1}{c} \frac{\partial(\mathbf{H}+4 \pi \mathbf{M})}{\partial t}, \\
\nabla \times \mathbf{H}=\frac{4 \pi \sigma}{c} \mathbf{E} .
\end{gathered}
$$

The high value of conductivity $\sigma$ allows us to neglect the displacement current compared to the conduction current. We restrict ourselves to the consideration of an in-plane magnetized film. The theory for the case of out-of-plane magnetization can be derived in a similar way. Then we follow the derivation presented in Ref. 27 (note that Ament and Rado wrote the damping term in the different form and also used different directions of the coordinate axes). After the linearization of the system [Eq. (A1)] and eliminating electric field $\mathbf{E}$, we arrive to the following equation:

$$
8 \pi i \tilde{m}_{y}=\delta^{2} \frac{\partial^{2} \tilde{h}_{y}}{\partial z^{2}}-2 i \tilde{h}_{y},
$$

where $\delta=\sqrt{\frac{c^{2}}{2 \pi \omega \sigma}}$ is a classical skin depth and $h_{y}$ is now the stray field from the magnetization rather than the incident uniform magnetic field. In our case, the first term on the right-hand side of the equation is about $\frac{\delta^{2}}{d^{2}} \approx 10^{7}-10^{8}$ greater than the other two terms, assuming conductivity $\sigma$ of either cobalt or iron of about $10^{14} \mathrm{~s}^{-1}$ at $100 \mathrm{GHz} .{ }^{40}$ The latter two terms can therefore be safely neglected in the present problem and only have to be taken into account for much thicker magnetic films. ${ }^{28,29}$ So, the eddy current and exchangeconductivity effects do not play a significant role in the present case.

\section{APPENDIX B: THE EXACT FORM OF THE SOLUTION FOR DIFFERENT BOUNDARY CONDITIONS}

Functions $\tilde{f}_{\perp, \|}, \tilde{p}_{\perp, \|}, \tilde{g}_{\perp, \|}$, and $\tilde{q}_{\perp, \|}$ from Eqs. (7a) and (7b) are given by

$$
\begin{gathered}
\tilde{f}_{\perp}=\frac{\eta+4 \pi+i \Omega \nu}{\eta^{2}-\Omega^{2}+4 \pi \eta+2 i \nu \Omega(\eta+2 \pi)} \\
\tilde{g}_{\perp}=-\frac{i \Omega}{\eta^{2}-\Omega^{2}+4 \pi \eta+2 i \nu \Omega(\eta+2 \pi)} \\
\tilde{p}_{\perp}=\frac{i \Omega}{\eta^{2}-\Omega^{2}+4 \pi \eta+2 i \nu \Omega(\eta+2 \pi)} \\
\tilde{q}_{\perp}=\frac{\eta+i \Omega \nu}{\eta^{2}-\Omega^{2}+4 \pi \eta+2 i \nu \Omega(\eta+2 \pi)} \\
\tilde{f}_{\|}=\frac{i \Omega}{\eta^{2}-\Omega^{2}+2 i \nu \Omega \eta} \\
\tilde{g}_{\|}=\frac{\eta+i \Omega \nu}{\eta^{2}-\Omega^{2}+2 i \nu \Omega \eta} \\
\tilde{p}_{\|}=\frac{\eta+i \Omega \nu}{\eta^{2}-\Omega^{2}+2 i \nu \Omega \eta} \\
\tilde{q}^{2}-\Omega^{2}+2 i \nu \Omega \eta
\end{gathered}
$$

The general boundary conditions [Eq. (8)] have two useful limiting cases. The first is the one of perfect pinning at both surfaces so that

$$
\begin{aligned}
\tilde{m}_{i} & =0, \quad \xi=0, \quad i=x, y, z . \\
\tilde{m}_{i} & =0, \quad \xi=l,
\end{aligned}
$$

Then, one can find that

$$
\begin{gathered}
C_{1}=\frac{\left(\tilde{f}_{\perp} \tilde{h}_{y}+\tilde{p}_{\perp} \tilde{h}_{z}\right)\left(\kappa_{\perp}^{2}-\eta-i \Omega \nu\right)\left(e^{-i k_{\perp} l}-1\right)+i \Omega\left(\tilde{g}_{\perp} \tilde{h}_{y}+\tilde{q}_{\perp} \tilde{h}_{z}\right)\left(e^{-i k_{\perp} l}-1\right)}{\left(k_{\perp}^{2}+\kappa_{\perp}^{2}\right)\left(e^{i k_{\perp} l}-e^{-i k_{\perp} l}\right)}, \\
C_{2}=-\frac{\left(\tilde{f}_{\perp} \tilde{h}_{y}+\tilde{p}_{\perp} \tilde{h}_{z}\right)\left(\kappa_{\perp}^{2}-\eta-i \Omega \nu\right)\left(e^{i k_{\perp} l}-1\right)+i \Omega\left(\tilde{g}_{\perp} \tilde{h}_{y}+\tilde{q}_{\perp} \tilde{h}_{z}\right)\left(e^{i k_{\perp} l}-1\right)}{\left(k_{\perp}^{2}+\kappa_{\perp}^{2}\right)\left(e^{i k_{\perp} l}-e^{-i k_{\perp} l}\right)}, \\
C_{3}=\frac{\left(\tilde{f}_{\perp} \tilde{h}_{y}+\tilde{p}_{\perp} \tilde{h}_{z}\right)\left(k_{\perp}^{2}+\eta+i \Omega \nu\right)\left(e^{-\kappa_{\perp} l}-1\right)-i \Omega\left(\tilde{g}_{\perp} \tilde{h}_{y}+\tilde{q}_{\perp} \tilde{h}_{z}\right)\left(e^{-\kappa_{\perp} l}-1\right)}{\left(k_{\perp}^{2}+\kappa_{\perp}^{2}\right)\left(e^{\kappa_{\perp} l}-e^{-\kappa_{\perp} l}\right)}, \\
C_{4}=-\frac{\left(\tilde{f}_{\perp} \tilde{h}_{y}+\tilde{p}_{\perp} \tilde{h}_{z}\right)\left(k_{\perp}^{2}+\eta+i \Omega \nu\right)\left(e^{\kappa_{\perp} l}-1\right)-i \Omega\left(\tilde{g}_{\perp} \tilde{h}_{y}+\tilde{q}_{\perp} \tilde{h}_{z}\right)\left(e^{\kappa_{\perp} l}-1\right)}{\left(k_{\perp}^{2}\right)\left(e^{\kappa_{\perp} l}-e^{-\kappa_{\perp} l}\right)}, \\
D_{1}=\frac{\left(\tilde{f}_{\|} \tilde{h}_{y}+\tilde{p}_{\|} \tilde{h}_{x}\right)\left(\kappa_{\|}^{2}-\eta-i \Omega \nu\right)\left(e^{-i k_{\|} l}-1\right)+i \Omega\left(\tilde{g}_{\|} \tilde{h}_{y}+\tilde{q}_{\|} \tilde{h}_{x}\right)\left(e^{-i k_{\|} l}-1\right)}{\left(k_{\|}^{2}\right)\left(e^{i k_{\|} l}-e^{-i k_{\|} l}\right)},
\end{gathered}
$$




$$
\begin{aligned}
& D_{2}=-\frac{\left(\tilde{f}_{\|} \tilde{h}_{y}+\tilde{p}_{\|} \tilde{h}_{x}\right)\left(\kappa_{\|}^{2}-\eta-i \Omega \nu\right)\left(e^{i k_{\|} l}-1\right)+i \Omega\left(\tilde{g}_{\|} \tilde{h}_{y}+\tilde{q}_{\|} \tilde{h}_{x}\right)\left(e^{i k_{\|} l}-1\right)}{\left(k_{\|}^{2}+\kappa_{\|}^{2}\right)\left(e^{i k_{\|} l}-e^{-i k_{\|} l}\right)}, \\
& D_{3}=\frac{\left(\tilde{f}_{\|} \tilde{h}_{y}+\widetilde{p}_{\|} \tilde{h}_{x}\right)\left(k_{\|}^{2}+\eta+i \Omega \nu\right)\left(e^{-\kappa_{\|} l}-1\right)-i \Omega\left(\widetilde{g}_{\|} \tilde{h}_{y}+\widetilde{q}_{\|} \tilde{h}_{x}\right)\left(e^{-\kappa_{\|} l}-1\right)}{\left(k_{\|}^{2}+\kappa_{\|}^{2}\right)\left(e^{\kappa_{\|} l}-e^{-\kappa_{\|} l}\right)}, \\
& D_{4}=-\frac{\left(\tilde{f}_{\|} \tilde{h}_{y}+\tilde{p}_{\|} \tilde{h}_{x}\right)\left(k_{\|}^{2}+\eta+i \Omega \nu\right)\left(e^{\kappa_{\|} l}-1\right)-i \Omega\left(\tilde{g}_{\|} \tilde{h}_{y}+\tilde{q}_{\|} \tilde{h}_{x}\right)\left(e^{\kappa_{\|} l}-1\right)}{\left(k_{\|}^{2}+\kappa_{\|}^{2}\right)\left(e^{\kappa_{\|} l}-e^{-\kappa_{\|} l}\right)} .
\end{aligned}
$$

The resonance frequencies can be found from condition

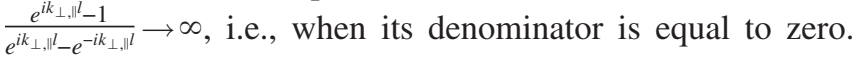
Here, $\kappa$ is not a wave vector but describes a nonresonant part of the solution. That is why we do not consider the part containing $e^{\kappa_{\perp, \|} l}-e^{-\kappa_{\perp, \|} l}$ in the denominator. The resonance values of wave vectors $k_{\perp}$ and $k_{\|}$are given by $k_{\perp, \|}=\frac{\pi(2 n+1)}{l}$, where $n=0,1,2,3, \ldots$ This conclusion is in agreement with Kittel's result from Ref. 26. The corresponding resonance frequencies can be found from Eq. (5).

The second case corresponds to free spins at one surface of each film and perfect pinning at the other so that

$$
\begin{aligned}
& \frac{\partial \tilde{m}_{i}}{\partial \xi}=0, \quad \xi=0, \quad i=x, y, z . \\
& \tilde{m}_{i}=0, \quad \xi=l,
\end{aligned}
$$

Then, we obtain

$$
\begin{gathered}
C_{1}=-\frac{\left(\tilde{f}_{\perp} \tilde{h}_{y}+\tilde{p}_{\perp} \tilde{h}_{z}\right)\left(\kappa_{\perp}^{2}-\eta-i \Omega \nu\right)+i \Omega\left(\tilde{g}_{\perp} \tilde{h}_{y}+\tilde{q}_{\perp} \tilde{h}_{z}\right)}{\left(k_{\perp}^{2}+\kappa_{\perp}^{2}\right)\left(e^{i k_{\perp} l}+e^{-i k_{\perp} l}\right)}, \\
C_{2}=C_{1}, \\
C_{3}=-\frac{\left(\tilde{f}_{\perp} \tilde{h}_{y}+\tilde{p}_{\perp} \tilde{h}_{z}\right)\left(k_{\perp}^{2}+\eta+i \Omega \nu\right)-i \Omega\left(\tilde{g}_{\perp} \tilde{h}_{y}+\tilde{q}_{\perp} \tilde{h}_{z}\right)}{\left(k_{\perp}^{2}+\kappa_{\perp}^{2}\right)\left(e^{\kappa_{\perp} l}+e^{-\kappa_{\perp} l}\right)}, \\
C_{4}=C_{3}, \\
D_{1}=-\frac{\left(\tilde{f}_{\|} \tilde{h}_{y}+\tilde{p}_{\|} \tilde{h}_{x}\right)\left(\kappa_{\|}^{2}-\eta-i \Omega \nu\right)+i \Omega\left(\tilde{g}_{\|} \tilde{h}_{y}+\tilde{q}_{\|} \tilde{h}_{x}\right)}{\left(k_{\|}^{2}+\kappa_{\|}^{2}\right)\left(e^{i k_{\|} l}+e^{-i k_{\|} l}\right)},
\end{gathered}
$$

$$
\begin{gathered}
D_{2}=D_{1}, \\
D_{3}=-\frac{\left(\tilde{f}_{\|} \tilde{h}_{y}+\tilde{p}_{\|} \tilde{h}_{x}\right)\left(k_{\|}^{2}+\eta+i \Omega \nu\right)-i \Omega\left(\tilde{g}_{\|} \tilde{h}_{y}+\tilde{q}_{\|} \tilde{h}_{x}\right)}{\left(k_{\|}^{2}+\kappa_{\|}^{2}\right)\left(e^{\kappa_{\|} l}+e^{-\kappa_{\|} l}\right)}, \\
D_{4}=D_{3} .
\end{gathered}
$$

In this case, the condition of resonance is $\frac{1}{e^{i k_{\perp, l}}+e^{-i k_{\perp, l l}}} \rightarrow \infty$. The resonant values of wave vectors $k_{\perp}$ and $k_{\|}$are $k_{\perp, \|}=\frac{\pi(n+1 / 2)}{l}$, where $n=0,1,2,3, \ldots$

It is also possible to obtain analytical solutions for the case of exchange boundary conditions of the general form [Eq. (8)] but the corresponding formulas are very long. So, we restrict ourselves to a simpler case, which is however applicable for many common experimental situations. We consider the case when spins are free at one side but are pinned at another side with a finite strength. The strength of the latter pinning is described in terms of the pinning parameter $\beta$ (see Sec. II). Then, the boundary conditions are

$$
\begin{aligned}
& \frac{\partial \tilde{m}_{i}}{\partial \xi}=0, \quad \xi=0, \\
& \frac{\partial \tilde{m}_{i}}{\partial \xi}+\beta \tilde{m}_{i}=0, \quad \xi=l,
\end{aligned} \quad i=x, y, z .
$$

The associated amplitudes are given by

$$
\begin{gathered}
C_{1}=-i \beta \frac{\left(\tilde{f}_{\perp} \tilde{h}_{y}+\tilde{p}_{\perp} \tilde{h}_{z}\right)\left(\kappa_{\perp}^{2}-\eta-i \Omega \nu\right)+i \Omega\left(\widetilde{g}_{\perp} \tilde{h}_{y}+\tilde{q}_{\perp} \tilde{h}_{z}\right)}{\left(k_{\perp}^{2}+\kappa_{\perp}^{2}\right)\left[i \beta\left(e^{i k_{\perp} l}+e^{-i k_{\perp} l}\right)-k_{\perp}\left(e^{i k_{\perp} l}-e^{-i k_{\perp} l}\right)\right]}, \\
C_{2}=C_{1},
\end{gathered}
$$

$$
\begin{gathered}
C_{3}=-\beta \frac{\left(\tilde{f}_{\perp} \tilde{h}_{y}+\tilde{p}_{\perp} \tilde{h}_{z}\right)\left[\left(e^{i k_{\perp} l}+e^{-i k_{\perp} l}\right)\left(i \beta \eta+i \beta k_{\perp}^{2}-\beta \Omega \nu\right)-\left(e^{i k_{\perp} l}-e^{-i k_{\perp} l}\right)\left(k_{\perp}^{3}+k_{\perp} \eta+i k_{\perp} \Omega \nu\right)\right]}{\left(k_{\perp}^{2}+\kappa_{\perp}^{2}\right)\left[i \beta\left(e^{i k_{\perp} l}+e^{-i k_{\perp} l}\right)-k_{\perp}\left(e^{i k_{\perp} l}-e^{-i k_{\perp} l}\right)\right]\left[\kappa_{\perp}\left(e^{\kappa_{\perp} l}-e^{-\kappa_{\perp} l}\right)+\beta\left(e^{\kappa_{\perp} l}+e^{-\kappa_{\perp} l}\right)\right]} \\
-\beta \frac{\left(\widetilde{g}_{\perp} \tilde{h}_{y}+\widetilde{q}_{\perp} \tilde{h}_{z}\right)\left[\beta \Omega\left(e^{i k_{\perp} l}+e^{-i k_{\perp} l}\right)-i k_{\perp} \Omega\left(e^{i k_{\perp} l}-e^{-i k_{\perp} l}\right)\right]}{\left(k_{\perp}^{2}+\kappa_{\perp}^{2}\right)\left[i \beta\left(e^{i k_{\perp} l}+e^{-i k_{\perp} l}\right)-k_{\perp}\left(e^{i k_{\perp} l}-e^{-i k_{\perp} l}\right)\right]\left[\kappa_{\perp}\left(e^{\kappa_{\perp} l}-e^{-\kappa_{\perp} l}\right)+\beta\left(e^{\kappa_{\perp} l}+e^{-\kappa_{\perp} l}\right)\right]}, \\
C_{4}=C_{3},
\end{gathered}
$$




$$
\begin{aligned}
& D_{1}=-i \beta \frac{\left(\tilde{f}_{\|} \widetilde{h}_{y}+\widetilde{p}_{\|} \tilde{h}_{x}\right)\left(\kappa_{\|}^{2}-\eta-i \Omega \nu\right)+i \Omega\left(\widetilde{g}_{\|} \widetilde{h}_{y}+\widetilde{q}_{\|} \widetilde{h}_{x}\right)}{\left(k_{\|}^{2}+\kappa_{\|}^{2}\right)\left[i \beta\left(e^{i k_{\|} l}+e^{-i k_{\|} l}\right)-k_{\|}\left(e^{i k_{\|} l}-e^{-i k_{\|} l}\right)\right]}, \\
& D_{2}=D_{1}, \\
& D_{3}=-\beta \frac{\left(\tilde{f}_{\|} \widetilde{h}_{y}+\widetilde{p}_{\|} \widetilde{h}_{x}\right)\left[\left(e^{i k_{\|} l}+e^{-i k_{\|} l}\right)\left(i \beta \eta+i \beta k_{\|}^{2}-\beta \Omega \nu\right)-\left(e^{i k_{\|} l}-e^{-i k_{\|} l}\right)\left(k_{\|}^{3}+k_{\|} \eta+i k_{\|} \Omega \nu\right)\right]}{\left(k_{\|}^{2}+\kappa_{\|}^{2}\right)\left[i \beta\left(e^{i k_{\|} l}+e^{-i k_{\|} l}\right)-k_{\|}\left(e^{i k_{\|} l}-e^{-i k_{\|} l}\right)\right]\left[\kappa_{\|}\left(e^{\kappa_{\|} l}-e^{-\kappa_{\|} l}\right)+\beta\left(e^{\kappa_{\|} l}+e^{-\kappa_{\|} l}\right)\right]} \\
& -\beta \frac{\left(\tilde{g}_{\|} \tilde{h}_{y}+\tilde{q}_{\|} \tilde{h}_{x}\right)\left[\beta \Omega\left(e^{i k_{\|} l}+e^{-i k_{\|} l}\right)-i k_{\|} \Omega\left(e^{i k_{\|} l}-e^{-i k_{\|} l}\right)\right]}{\left(k_{\|}^{2}+\kappa_{\|}^{2}\right)\left[i \beta\left(e^{i k_{\|} l}+e^{-i k_{\|} l}\right)-k_{\|}\left(e^{i k_{\|} l}-e^{-i k_{\|} l}\right)\right]\left[\kappa_{\|}\left(e^{\kappa_{\|} l}-e^{-\kappa_{\|} l}\right)+\beta\left(e^{\kappa_{\|} l}+e^{-\kappa_{\|} l}\right)\right]}, \\
& D_{4}=D_{3} .
\end{aligned}
$$

\section{APPENDIX C: DERIVATION OF THE MACROSCOPIC EFFECTIVE PERMEABILITY OF THE METAMATERIAL}

To find the macroscopic magnetization entering the Maxwell equations, we average the excited microscopic magnetization over the infinitesimal interval as follows:

$$
\widetilde{\mathbf{m}}^{(\text {macro })}(\Omega)=\frac{1}{L} \int_{0}^{L} \widetilde{\mathbf{m}}^{(\text {micro })}(\xi, \Omega) d \xi,
$$

of integration, and $N$ is the number of those films. Since the films are identical and do not interact with each other we can rewrite Eq. (C1) in the form

$$
\widetilde{\mathbf{m}}^{(\text {macro })}(\Omega)=\frac{N}{L} \int_{0}^{l} \widetilde{\mathbf{m}}(\xi, \Omega) d \xi=\rho \frac{1}{l} \int_{0}^{l} \widetilde{\mathbf{m}}(\xi, \Omega) d \xi,
$$

where $\widetilde{\mathbf{m}}^{(\text {micro })}=\Sigma_{N} \widetilde{\mathbf{m}}_{N}(\xi-N l, \Omega)$ and $L=\frac{\Lambda}{\sqrt{\alpha}}$. Here, $\widetilde{\mathbf{m}}_{N}(\xi$ $-N l, \Omega$ ) denotes the magnetization in the form of Eqs. (7a) and (7b) of the $N$ th film, which is situated inside the interval where $\rho=\frac{N l}{L}=\frac{N d}{\Lambda}$ is the filling fraction of the films and

$$
\begin{aligned}
& \frac{1}{l} \int_{0}^{l} \widetilde{m}_{y}(\xi, \Omega) d \xi=\frac{C_{1}}{i k_{\perp} l}\left(e^{i k_{\perp} l}-1\right)-\frac{C_{2}}{i k_{\perp} l}\left(e^{-i k_{\perp} l}-1\right)+\frac{C_{3}}{\kappa_{\perp} l}\left(e^{\kappa_{\perp} l}-1\right)-\frac{C_{4}}{\kappa_{\perp} l}\left(e^{-\kappa_{\perp} l}-1\right)+\tilde{f}_{\perp} \tilde{h}_{y}+\widetilde{p}_{\perp} \tilde{h}_{z}, \\
& \frac{1}{l} \int_{0}^{l} \tilde{m}_{z}(\xi, \Omega) d \xi=\frac{\eta+i \Omega \nu+k_{\perp}^{2}}{i \Omega}\left[\frac{C_{1}}{i k_{\perp} l}\left(e^{i k_{\perp} l}-1\right)-\frac{C_{2}}{i k_{\perp} l}\left(e^{-i k_{\perp} l}-1\right)\right]+\frac{\eta+i \Omega \nu-\kappa_{\perp}^{2}}{i \Omega}\left[\frac{C_{3}}{\kappa_{\perp} l}\left(e^{\kappa_{\perp} l}-1\right)-\frac{C_{4}}{\kappa_{\perp} l}\left(e^{-\kappa_{\perp} l}-1\right)\right] \\
& +\widetilde{g}_{\perp} \tilde{h}_{y}+\widetilde{q}_{\perp} \tilde{h}_{z}, \\
& \frac{1}{l} \int_{0}^{l} \tilde{m}_{x}(\xi, \Omega) d \xi=\frac{D_{1}}{i k_{\|} l}\left(e^{i k_{\|} l}-1\right)-\frac{D_{2}}{i k_{\|} l}\left(e^{-i k_{\|} l}-1\right)+\frac{D_{3}}{\kappa_{\|} l}\left(e^{\kappa_{\|} l}-1\right)-\frac{D_{4}}{\kappa_{\|} l}\left(e^{-\kappa_{\|} l}-1\right)+\tilde{f}_{\|} \tilde{h}_{y}+\tilde{p}_{\|} \tilde{h}_{x}, \\
& \frac{1}{l} \int_{0}^{l} \widetilde{m}_{y}(\xi, \Omega) d \xi=\frac{\eta+i \Omega \nu+k_{\|}^{2}}{i \Omega}\left[\frac{D_{1}}{i k_{\perp} l}\left(e^{i k_{\perp} l}-1\right)-\frac{D_{2}}{i k_{\perp} l}\left(e^{-i k_{\perp} l}-1\right)\right]+\frac{\eta+i \Omega \nu-\kappa_{\|}^{2}}{i \Omega}\left[\frac{D_{3}}{\kappa_{\|} l}\left(e^{\kappa_{\|} l}-1\right)-\frac{D_{4}}{\kappa_{\|} l}\left(e^{-\kappa_{\|} l}-1\right)\right]+\widetilde{g}_{\|} \widetilde{h}_{y}+\widetilde{q}_{\|} \widetilde{h}_{x} .
\end{aligned}
$$

Using Eqs. (C1) and (C2) and formulas for the amplitudes $C_{1}, C_{2}, C_{3}, C_{4}$ and $D_{1}, D_{2}, D_{3}, D_{4}$ (see Appendix B), we can write the macroscopic magnetization in cases of in-plane and out-of-plane magnetizations through the susceptibility tensor components, 


$$
\begin{aligned}
& \tilde{m}_{y}^{(\text {macro })}=\chi_{\perp y y} \tilde{h}_{y}+\chi_{\perp y z} \tilde{h}_{z}, \\
& \tilde{m}_{z}^{(\text {macro })}=\chi_{\perp z y} \tilde{h}_{y}+\chi_{\perp z z} \tilde{h}_{z},
\end{aligned}
$$

and

$$
\tilde{m}_{x}^{(\text {macro })}=\chi_{\| x x} \tilde{h}_{x}+\chi_{\| x y} \tilde{h}_{y},
$$

$$
\tilde{m}_{y}^{(\text {macro })}=\chi_{\| x y} \tilde{h}_{x}+\chi_{\| y y} \tilde{h}_{y} .
$$

The exact form of the susceptibility tensor components is determined by the form of the boundary conditions. However, in each case $\chi_{\perp z y}=-\chi_{\perp y z}, \chi_{\| y x}=-\chi_{\| x y}$, and $\chi_{\| x x}=\chi_{\| y y}$.

The effective permeability tensor is then defined as $\hat{\mu}_{\perp, \|}$ $=\hat{1}+4 \pi \hat{\chi}_{\perp, \|}$. Using the latter identity, one can obtain the tensors used in Eqs. (11a) and (11b). *rm350@exeter.ac.uk

†v.v.kruglyak@exeter.ac.uk

${ }^{1}$ V. G. Veselago, Sov. Phys. Usp. 10, 509 (1968).

${ }^{2}$ J. B. Pendry, Phys. Rev. Lett. 85, 3966 (2000).

${ }^{3}$ J. B. Pendry, D. Schurig, and D. R. Smith, Science 312, 1780 (2006).

${ }^{4}$ V. M. Shalaev, Nat. Photonics 1, 41 (2007).

${ }^{5}$ J. B. Pendry, A. J. Holden, D. J. Robbins, and W. J. Stewart, IEEE Trans. Microwave Theory Tech. 47, 2075 (1999).

${ }^{6}$ D. R. Smith, W. J. Padilla, D. C. Vier, S. C. Nemat-Nasser, and S. Schultz, Phys. Rev. Lett. 84, 4184 (2000).

${ }^{7}$ R. A. Shelby, D. R. Smith, and S. Schultz, Science 292, 77 (2001).

${ }^{8}$ S. Zhang, W. Fan, B. K. Minhas, A. Frauenglass, K. J. Malloy, and S. R. J. Brueck, Phys. Rev. Lett. 94, 037402 (2005).

${ }^{9}$ S. Zhang, W. Fan, K. J. Malloy, and S. R. J. Brueck, J. Opt. Soc. Am. B 23, 434 (2006).

${ }^{10}$ O. Acher, J. Magn. Magn. Mater. 321, 2093 (2009).

${ }^{11}$ A. G. Gurevich and G. A. Melkov, Magnetization Oscillations and Waves (CRC Press, Cleveland, 1996).

${ }^{12}$ S. V. Vasiliev, V. V. Kruglyak, M. L. Sokolovskii, and A. N. Kuchko, J. Appl. Phys. 101, 113919 (2007).

${ }^{13}$ V. V. Kruglyak, S. O. Demokritov, and D. Grundler, J. Phys. D: Appl. Phys. 43, 264001 (2010).

${ }^{14}$ When our manuscript was under review, we learned about results from P. Buczek, A. Ernst, and L. M. Sandratskii, Phys. Rev. Lett. 105, 097205 (2010). In this reference, the authors could not offer a mechanism of excitation of $\mathrm{THz}$ spin waves, in contrast to the present work where the coupling of the uniform magnetic field to nonuniform spin waves is facilitate by the surface pinning.

${ }^{15}$ U. Ebels, J. L. Duvail, P. E. Wigen, L. Piraux, L. D. Buda, and K. Ounadjela, Phys. Rev. B 64, 144421 (2001).

${ }^{16}$ G. S. Makeeva, M. Pardavi-Horvath, and O. A. Golovanov, IEEE Trans. Magn. 45, 4074 (2009).

${ }^{17}$ V. Boucher, L.-P. Carignan, T. Kodera, C. Caloz, A. Yelon, and D. Ménard, Phys. Rev. B 80, 224402 (2009).

${ }^{18}$ S. Rajagopalan and J. K. Furdyna, Phys. Rev. B 39, 2532 (1989).

${ }^{19}$ A. Aharoni, J. Appl. Phys. 69, 7762 (1991); 81, 830 (1997).

${ }^{20} \mathrm{G}$. Viau, F. Fievet-Vincent, F. Fievet, P. Toneguzzo, F. Ravel, and O. Acher, J. Appl. Phys. 81, 2749 (1997).

${ }^{21}$ D. Mercier, J.-C. S. Levy, G. Viau, F. Fievet-Vincent, F. Fievet, P. Toneguzzo, and O. Acher, Phys. Rev. B 62, 532 (2000).

${ }^{22}$ J. Ramprecht and D. Sjöberg, J. Phys. D: Appl. Phys. 41, 135005 (2008).
${ }^{23}$ L. Landau and E. Lifshitz, Phys. Z. Sowjetunion 8, 153 (1935).

${ }^{24}$ J. H. E. Griffiths, Nature (London) 158, 670 (1946).

${ }^{25}$ N. Bloembergen, Phys. Rev. 78, 572 (1950).

${ }^{26}$ C. Kittel, Phys. Rev. 110, 1295 (1958).

${ }^{27}$ W. S. Ament and G. T. Rado, Phys. Rev. 97, 1558 (1955).

${ }^{28}$ P. Pincus, Phys. Rev. 118, 658 (1960).

${ }^{29}$ J. M. Lock, Br. J. Appl. Phys. 17, 1645 (1966).

${ }^{30}$ L. L. Hirst, Phys. Rev. 164, 971 (1967).

${ }^{31}$ C. Vittoria, G. C. Bailey, R. C. Barker, and A. Yelon, Phys. Rev. B 7, 2112 (1973).

${ }^{32}$ T. G. Phillips, Proc. R. Soc. London, Ser. A 292, 224 (1966).

${ }^{33}$ F. Schreiber and Z. Frait, Phys. Rev. B 54, 6473 (1996).

${ }^{34}$ R. N. Nosov and D. I. Sementsov, Fiz. Tverd. Tela (St. Petersburg) 43, 1845 (2001) [Phys. Solid State 43, 1923 (2001)].

${ }^{35}$ R. J. Hicken, A. Barman, V. V. Kruglyak, and S. Ladak, J. Phys. D: Appl. Phys. 36, 2183 (2003).

${ }^{36}$ A. Martins, S. C. Trippe, A. D. Santos, and F. Pelegrini, J. Magn. Magn. Mater. 308, 120 (2007).

${ }^{37}$ A. D. C. Viegas, M. A. Corrêa, L. Santi, R. B. da Silva, F. Bohn, M. Carara, and R. L. Sommer, J. Appl. Phys. 101, 033908 (2007).

${ }^{38}$ G. C. Bailey and C. Vittoria, Phys. Rev. Lett. 28, 100 (1972).

${ }^{39}$ C. Vittoria, Phys. Rev. B 32, 1679 (1985).

${ }^{40}$ M. A. Ordal, L. L. Long, R. J. Bell, S. E. Bell, R. R. Bell, R. W. Alexander, Jr., and C. A. Ward, Appl. Opt. 22, 1099 (1983).

${ }^{41}$ W. J. Padilla, A. J. Taylor, C. Highstrete, M. Lee, and R. D. Averitt, Phys. Rev. Lett. 96, 107401 (2006).

${ }^{42}$ H.-T. Chen, J. F. O'Hara, A. K. Azad, A. J. Taylor, R. D. Averitt, D. B. Shrekenhamer, and W. J. Padilla, Nat. Photonics 2, 295 (2008).

${ }^{43}$ T. J. Yen, W. J. Padilla, N. Fang, D. C. Vier, D. R. Smith, J. B. Pendry, D. N. Basov, and X. Zhang, Science 303, 1494 (2004).

${ }^{44}$ W. J. Padilla, D. R. Smith, and D. N. Basov, J. Opt. Soc. Am. B 23, 404 (2006).

${ }^{45}$ H.-T. Chen, W. J. Padilla, J. M. O. Zide, A. C. Gossard, A. J. Taylor, and R. D. Averitt, Nature (London) 444, 597 (2006).

${ }^{46}$ R. W. Boyd, R. J. Gehr, G. L. Fischer, and J. E. Sipe, Pure Appl. Opt. 5, 505 (1996).

${ }^{47}$ J. Gu, J. Han, X. Lu, R. Singh, Z. Tian, Q. Xing, and W. Zhang, Opt. Express 17, 20307 (2009).

${ }^{48}$ P. Weis, O. Paul, C. Imhof, R. Beigang, and M. Rahm, Appl. Phys. Lett. 95, 171104 (2009).

${ }^{49}$ R. H. Tarkhanyan, D. G. Niarchos, and M. Kafesaki, J. Magn. Magn. Mater. 322, 603 (2010).

${ }^{50}$ C. Menzel, T. Paul, C. Rockstuhl, T. Pertsch, S. Tretyakov, and F. Lederer, Phys. Rev. B 81, 035320 (2010). 